\title{
Caracterización técnico-administrativa de los sistemas de producción bovino de leche de pequeña escala en una región central de Colombia
}

\author{
Technical and administrative characterization of small-holder milk production \\ systems in a central region of Colombia
}

Alexander Nivia ${ }^{1 *}$; Edwin Beltrán ${ }^{1}$; Diana Marentes ${ }^{2}$; Andrés Pineda ${ }^{3}$

\begin{abstract}
RESUMEN
El objetivo de este trabajo fue realizar la caracterización técnico-administrativa de los sistemas de producción bovino de leche de pequeña escala ubicados en la vereda El Peñón, (municipio de Sibaté/Colombia). El estudio se llevó a cabo durante los meses de noviembre/2016 a abril/2017. La información fue obtenida a través de entrevistas semiestructuradas con 18 productores. Para la identificación de los sistemas se indagó variables técnico-productivas y la caracterización incluyó el análisis de los componentes: a) Localización, aspectos biofísicos, paisaje, uso y manejo de la tierra; b) Recurso forrajero y alimentación; c) Recurso animal; d) Reproducción y salud; e) Producción de leche, comercialización pecuaria y gestión empresarial; f) Mano de obra, infraestructura y equipos; y g) Información propietario. Se realizó un análisis de conglomerados y correspondencias múltiples utilizando el paquete estadístico SAS (Statistical analysis system, versión 9,4). Los resultados del análisis de conglomerados mostraron la conformación de 4 grupos clasificados como: lechería especializada, semiespecializada, pequeña escala y familiar dada su heterogeneidad del modelo productivo. Se identificaron 5 sistemas de pequeña escala y el análisis de correspondencias múltiples mostró relación entre variables cualitativas y componentes evidenciando un modelo tecnológico basado en una producción semiintensiva con adecuación de infraestructura y adquisición de tecnología de bajo costo con bajas estrategias de alimentación validadas. Utilización de recurso animal de baja calidad genética con reducida implementación de programas de mejoramiento y biotecnologías reproductivas y planes sanitarios y mínimas prácticas de bienestar animal y buenas prácticas de ordeño. Adicionalmente, limitadas redes de comercialización y estrategias de gestión empresarial. En conclusión, los sistemas se desarrollan en condiciones heterogéneas, lo que obedece a la formulación de diversos modelos productivos. La caracterización de los sistemas de pequeña escala es necesaria para identificar recomendaciones a nivel tecnológico y la intervención de políticas para el mejoramiento y fortalecimiento de los sistemas productivos.
\end{abstract}

Palabras Claves: Producción de leche, sistemas pecuarios, análisis de correspondencias múltiples.

\begin{abstract}
The objective of this work was to carry out the technical-administrative characterization of smallholder of milk production systems located in the village of El Peñon, in the town of Sibaté/Colombia. The study was carried out during the months of November/2016 to April/2017. The information was obtained through semi-structured interviews with 18 producers. For the identification of the systems, technical-productive variables were investigated and the characterization included the analysis of components: a) Location, biophysical aspects, landscape, use and land management; b) Forage resources and food; c) Animal resources; d) Reproduction and health; e) Milk production, livestock marketing and business management; f) Handing, infrastructure and equipment; and g) Owner information. A cluster analysis and multiple correspondences analysis using the statistical package SAS (Statistical analysis system, version 9.4). The results of the cluster analysis were shown the conformation of 8 groups classified as: specialized dairy, semispecialized, smallholder and small farm given to heterogeneity of the productive model. Five smallholder systems were identified, the analysis of multiple correspondences showed a relationship between qualitative variables and components evidencing a technological model based on a semi-intensive production with adequate infrastructure and acquisition of low cost technology with low validated feeding strategies. The use of low genetic quality animal resources with reduced implementation of breeding programs and reproductive biotechnologies and healthy plans and the lack of practices of animal welfare and good milking practices. Additionally, limited marketing networks and business management strategies. In conclusion, the systems are developed in heterogeneous conditions, which is due to the formulation of different production models. The characterization of smallholder systems is necessary to identify technological recommendations and the intervention of policies for the improvement and strengthening of productive systems.
\end{abstract}

Key Words: Milk production, livestock systems, multiple correspondence analysis.

1 Universidad Santo Tomas, Facultad de Ciencias y Tecnologías - VUAD, Programa de Administración de Empresas Agropecuarias. Bogotá, Colombia.

2 Universidad de Ciencias Aplicadas y Ambientales - U.D.C.A., Facultad de Ingeniería, Programa de Ingeniería Agronómica. Bogotá, Colombia.

3 Secretaria de Agricultura, Desarrollo y Medio Ambiente, Alcaldía Municipal de Sibaté, Colombia.

* Autor por correspondencia: alexandernivia@ustadistancia.edu.co

Fecha de Recepción: 11 marzo, 2017.

Fecha de Aceptación: 19 mayo, 2018.

DOI: http://dx.doi.org/10.4067/S0718-34292018005000601. Publicado en línea: 23-agosto-2018. 


\section{Introducción}

La leche en Colombia ocupa un lugar importante dentro de la economía nacional como producto prioritario por la importancia que ofrece como alimento básico de la población, lo cual ha influenciado para que la producción de leche bovina se haya desarrollado en diferentes regiones del territorio nacional (Balcázar, 1992; CORPOICA, 1998; Murgueitio \& Calle, 1998; Murgueitio, 1999). Para el año 2014 se produjo alrededor de 5.902 millones de litros anuales de leche proveniente de diferentes sistemas de producción bovina (CNA, 2016), lo que ha permitido de forma histórica alcanzar niveles de autoabastecimiento del $98 \%$ (FEDEGAN, 2017; Ríos \& Gómez, 2008). Sin embargo, durante los últimos años se ha registrado un incremento en las importaciones del producto lácteo para cubrir la demanda de la población y la industria transformadora, dado al crecimiento de los contingentes negociados en los diferentes tratados de libre comercio y problemáticas ambientales (FEDEGAN, 2015). Así mismo, se ha registrado una reducción en el consumo per cápita, siendo de 143 litros en el año 2015 y reportando una caída del 2,2\% para el 2016 (CNA, 2016).

Diversos autores clasifican los sistemas de producción de leche como: lechería tropical o doble propósito, lechería intensiva y lechería en pequeña escala o campesina familiar (Martínez et al., 2012; Rodríguez et al., 2012; Hernández et al., 2013); lechería especializada de altura, de bajura y doble propósito (González-Echevarría et al., 2012; VargasLeiton et al., 2013); y lechería especializada, semiespecializada, familiar y doble propósito (CortezArriola et al., 2015). Por otra parte, en Colombia se tipifican en sistemas de lechería especializada y de doble propósito generalmente dada la existencia de sistemas heterogéneos y diversidad de condiciones agroecológicas, climatológicas y modelos de producción encontradas a nivel de trópico alto y bajo (CORPOICA, 1998; Calderón et al., 2006).

Específicamente, la región del Sumapáz se caracteriza por poseer sistemas de producción lechera y cárnica basado en la utilización de animales de la especie Bos taurus e indicus y sus cruces (Giraldo, 2008). No obstante, en especial el municipio de Sibaté cuenta con un inventario de biotipos raciales con orientación productiva hacia los sistemas de producción de leche, en el que se identifican los sistemas especializados, de doble propósito y pequeña escala; siendo este último, importante dentro de la economía local y regional por el relevante número de productores. Más aún, no se reporta información de caracterización de los sistemas de producción en la región de estudio.

La caracterización identifica variables que inciden en el grado de heterogeneidad y homogeneidad existente en los sistemas de producción, lo que permite obtener grupos representativos de subsistemas productivos (Solano et al., 2000; Smith et al., 2002; Solano et al., 2003; Valerio-Cabrera et al., 2004; Avilez et al., 2010; Martínez-García et al., 2012; Vargas-Leiton et al., 2013).

Por lo anterior, el objetivo del presente estudio fue caracterizar los sistemas de producción de leche de pequeña escala de la vereda El Peñón del municipio de Sibaté (Cundinamarca-Colombia), respecto a sus características estructurales, productivas y económicas.

\section{Materiales y Métodos}

\section{Área de estudio}

La investigación se llevó a cabo en la vereda El Peñón del municipio de Sibaté (Cundinamarca) ubicada geográficamente en las coordenadas $4^{\circ} 30^{\prime} 12^{\prime \prime} \mathrm{N}$ y $74^{\circ} 20^{\prime} 47^{\prime \prime} \mathrm{O}$, a una altura de 2767 $\mathrm{msnm}$. Es caracterizada por presentar un clima frio, con una temperatura media anual de $13,5^{\circ} \mathrm{C}$ y una precipitación pluvial de $723 \mathrm{~mm}$. Esta región se caracteriza por poseer una vocación agropecuaria dedicada especialmente a la lechería y el cultivo de fresa.

\section{Identificación de los sistemas de producción de leche}

El estudio se llevó a cabo durante los meses de noviembre de 2016 a abril de 2017. El método de obtención de la información de los sistemas de producción de leche fue a través de entrevistas semi-estructuradas y charlas informales con 18 productores, en el que se incluyó variables técnicas y productivas como: propiedad y extensión de la tierra, biotipo racial e inventario animal, modelo tecnológico de producción (tipo de sistema, alimentación y ordeño, producción de leche y almacenamiento), infraestructura, maquinaria y equipos, mano de obra y comercialización. 
Caracterización de los sistemas de producción de leche a pequeña escala

Para la recolección de la información de caracterización se aplicó una encuesta que indagó acerca de los siguientes componentes: a) Localización de la unidad productiva (propia, arriendo y área), aspectos biofísicos (uso actual de la tierra), paisaje, uso y manejo de la tierra (topografía, suelo, disponibilidad de agua, cultivos); b) Recurso forrajero (uso y manejo, métodos de siembra, fertilización, labores culturales) y alimentación (suplementos alimenticios); c) Recurso animal (inventario, información); d) Reproducción (tipo, indicadores reproductivos) y salud (manejo, control parásitos, medidas preventivas); e) Producción de leche (producción y destino, ordeño), comercialización pecuaria (producto) y gestión empresarial (registros, toma de decisiones, servicios); f) Mano de obra (recurso humano, costo) e infraestructura y equipos (descripción); y g) Información propietario (actividad). Las variables cualitativas y cuantitativas fueron codificadas para su análisis (Tabla 1).

\section{Análisis estadístico}

La información recolectada fue registrada en una hoja electrónica de Microsoft Excel (2010) para su organización y control. La base de datos correspondiente a la identificación de los sistemas de producción de leche fue procesada estadísticamente mediante un análisis multivariado (conglomerado) para su agrupación y clasificación. Para la caracterización de los sistemas de producción de leche a pequeña escala, las variables cualitativas y cuantitativas fueron evaluadas mediante un análisis de correspondencias múltiples y descriptivo utilizando el paquete estadístico SAS (Statistical analysis system, versión 9,4).

\section{Resultados y Discusión}

La información obtenida en las encuestas realizadas a los productores permitió la identificación de diferentes tipos de sistemas de producción de leche de acuerdo a sus características estructurales, técnicas y productivas. Los resultados del análisis de conglomerados permitieron clasificar los sistemas de producción en 4 grupos por su similitud en el análisis de las variables analizadas (Figura 1).

Los conglomerados que conformaron los sistemas de producción bovino de leche permitieron identificar modelos productivos heterogéneos debido a sus diversas condiciones de producción como a los variados tamaños de las unidades productivas. No obstante, dado el bajo número de sistemas de producción presentes en la región de estudio y a su diverso modelo productivo, se logró construir una tipología o clasificación en cuatro grupos descritos principalmente como: lechería especializada (28\%), lechería semiespecializada (22\%), lechería de pequeña escala (28\%) y lechería familiar (22\%). Dicha clasificación difiere con la reportada por Calderón, et al. (2006) y CORPOICA (1998), ya que ésta refleja la diversidad que existe en los sistemas de producción representado por la variación en las características relacionadas con el uso de tecnologías, manejo del recurso forrajero, suplementos y subproductos alimenticios, genética del recurso animal, infraestructura y mano de obra.

Para la caracterización de los sistemas de producción bovino de leche a pequeña escala descritos en el clúster (Figura 1), se realizó el análisis de las variables para la identificación del modelo de producción. Es de anotar, que no existen reportes de las variables estudiadas en la región de estudio; por lo que, la utilización del análisis de correspondencias múltiples permite la identificación de variables próximas entre sí que demuestra el grado de frecuencia de ocurrencia de dichas características correlacionadas en la población (Smith et al., 2002; Betancourt et al., 2005; Carrillo et al., 2011). Por lo anterior, los resultados obtenidos en este estudio se describen por componente.

Las unidades productivas están localizadas a $15 \mathrm{~km}$ del perímetro urbano con vías de acceso carreteables. Los sistemas cuentan con una extensión promedio de $9,15 \mathrm{Ha}$, distribuidas topográficamente en plana (15\%) y ondulada (85\%). En cuanto a los aspectos biofísicos, los sistemas no presentan áreas inundables, el tipo de suelo que predomina es franco arenoso y los nacederos y acueducto son la principal fuente de agua. El uso y distribución de la tierra se encuentran fraccionados en praderas (89\%), agricultura (4\%), bosque (5\%) y $2 \%$ para instalaciones (Figura 2a). Por lo cual, estas condiciones topográficas han favorecido el desarrollo de sistemas de producción ganadera principalmente la bovina. Ya que como lo reporta la FAO (2002), dada su ubicación geográfica privilegiada en la franja tropical contabiliza la mayor parte de los recursos forrajeros y ganaderos favoreciendo la implementación y desarrollo de estos sistemas productivos. 
Tabla 1. Variables consideradas para el análisis de correspondencias múltiples.

\begin{tabular}{|c|c|c|c|}
\hline \multicolumn{4}{|c|}{ a. Localización de la unidad productiva } \\
\hline $\mathrm{P}$ & Propia & OOtr & Ocupación Otros (Ha) \\
\hline APHa & Área Propia (Ha) & ANoIn & Áreas no inundables \\
\hline A & Arriendo & \multicolumn{2}{|c|}{ Paisaje, uso y manejo de la tierra } \\
\hline АAHa & Área Arriendo (Ha) & TPlanha & Topografía Plana (Ha) \\
\hline ACarr & Acceso carreteable & TOndha & Topografía Ondulada (Ha) \\
\hline \multicolumn{2}{|c|}{ Aspectos biofísicos } & TSue & Tipo suelo Franco Arenoso \\
\hline OAgr & Ocupación Agricultura (Ha) & ANac & Agua Nacedero \\
\hline OPra & Ocupación Praderas (Ha) & AAcue & Agua Acueducto \\
\hline Oras & Ocupación Rastrojo (Ha) & CPapa & Cultivo Papa \\
\hline OBos & Ocupación Bosque (Ha) & CQuin & Cultivo Quinua \\
\hline OIns & Ocupación Instalaciones (Ha) & & \\
\hline \multicolumn{4}{|c|}{ b. $\quad$ Recurso forrajero } \\
\hline PKVord & Kykuyo Vacas Ordeño & ATri & Abona Triple Quince \\
\hline PKVhor & Kykuyo Vacas Horras & Acal & Abona Cal Viva \\
\hline PKTer & Kykuyo Vacas Terneros & ABov & Abona Bovinaza \\
\hline PKAniC & Kykuyo Animales Crecimiento & AGal & Abona Gallinaza \\
\hline PKTor & Kykuyo Toros & ACom & Abona Compost \\
\hline PKPRot & Kykuyo Pastoreo Rotacional & TDesInv & Tiempo potreros Descanso Invierno \\
\hline PTRVord & Trébol Rojo Vacas Ordeño & TDesVer & Tiempo potreros Descanso Verano \\
\hline PTRVhor & Trébol Rojo Vacas Horras & TOcuInv & Tiempo potreros Ocupación Invierno \\
\hline PTRTer & Trébol Rojo Vacas Terneros & TOcuVer & Tiempo potreros Ocupación Verano \\
\hline PTRAniC & Trébol Rojo Animales Crecimiento & LCRast & Labores culturales Rastrillo \\
\hline PTRTor & Trébol Rojo Toros & LCAboQ & Labores culturales Abono Químico \\
\hline PTRPRot & Trébol Rojo Pastoreo Rotacional & LCAboO & Labores culturales Abono Orgánico \\
\hline PTBVord & Trébol Blanco Vacas Ordeño & LCConM & Labores culturales Control Malezas \\
\hline PTBVhor & Trébol Blanco Vacas Horras & LCRenP & Labores culturales Renovación Praderas \\
\hline PTBTer & Trébol Blanco Vacas Terneros & LCConP & Labores culturales Control Plagas \\
\hline PTBAniC & Trébol Blanco Animales crecimiento & DPViv & División potreros cerca Viva \\
\hline PTBTor & Trébol Blanco Toros & DVEle & División potreros cerca Eléctrica \\
\hline PTBPRot & Trébol Blanco Pastoreo Rotacional & DVPos & División potreros Postes madera \\
\hline PRVord & Raygrass Vacas Ordeño & AFSau & Árboles Forrajeros Sauco \\
\hline PRVhor & Raygrass Vacas Horras & AFAca & Árboles Forrajeros Acacia \\
\hline PRTer & Raygrass Vacas Terneros & AFSac & Árboles Forrajeros Sauce \\
\hline PRAniC & Raygrass Animales Crecimiento & AFAli & Árboles Forrajeros Aliso \\
\hline PRTor & Raygrass Toros & AFRam & Árboles Forrajeros Ramoneo \\
\hline PRPRot & Raygrass Pastoreo Rotacional & $\mathrm{AfCoS}$ & Árboles Forrajeros Corte y Suministro \\
\hline PAVord & Azul Orchoro Vacas Ordeño & \multicolumn{2}{|c|}{ Alimentación } \\
\hline PAVhor & Azul Orchoro Vacas Horras & SAli & Suministra otro tipo Alimentos \\
\hline PATer & Azul Orchoro Vacas Terneros & SVCon & Suministra Concentrado \\
\hline PAAniC & Azul Orchoro Animales Crecimiento & SVEns & Suministra Ensilaje \\
\hline PATor & Azul Orchoro Toros & SVPco & Suministra Productos de cosecha \\
\hline PAPRot & Azul Orchoro Pastoreo Rotacional & SVMel & Suministra Melaza \\
\hline GraAve & Gramíneas Avena & SVSal & Suministra Sal \\
\hline GraSiMec & Gramíneas Siembra Mecánico & FSPIn & Frecuencia Suministro Permanente Invierno \\
\hline
\end{tabular}


Continuación Tabla 1

\begin{tabular}{|c|c|c|c|}
\hline Abon & Abona & FSPVe & Frecuencia Suministro Permanente Verano \\
\hline \multirow[t]{2}{*}{ AUre } & Abona Urea & FSOIn & Frecuencia Suministro Ocasional Invierno \\
\hline & & FSOVe & Frecuencia Suministro Ocasional Verano \\
\hline \multicolumn{4}{|c|}{ Recurso animal } \\
\hline RNor & Raza Normando & $\mathrm{N} 12 \mathrm{a}$ & Novillos 1 - 2 años \\
\hline $\mathrm{RNxJ}$ & Raza Normando x Jersey & N23a & Novillos 2 -3 años \\
\hline RHol & Raza Holstein & ITIde & Identifica Terneras \\
\hline RJer & Raza Jersey & ITFNa & Identifica Terneras Fecha Nacimiento \\
\hline RAyr & Raza Ayrshire & ITPDe & Identifica Terneras Peso Destete \\
\hline VHor & Vacas Horras & ITPad & Identifica Terneras Padres \\
\hline $\mathrm{V} 1 \mathrm{p}$ & Vacas Primer parto & IVIde & Identifica Vacas \\
\hline V24p & Vacas 2 - 4 parto & IVPrL & Identifica Vacas Producción leche /Lactancia \\
\hline $\mathrm{V} 4 \mathrm{p}$ & Vacas $>4$ parto & IVFPa & Identifica Vacas Fecha Parto \\
\hline $\mathrm{N} 12 \mathrm{a}$ & Novillas $1-2$ años & IVFNa & Identifica Vacas Fecha Nacimiento \\
\hline $\mathrm{N} 23 \mathrm{a}$ & Novillas $2-3$ años & IVNSe & Identifica Vacas No. Servicios/Parto \\
\hline $\mathrm{T} 212 \mathrm{~m}$ & Terneras $2-12$ meses & IVDPa & Identifica Vacas Dificultad Parto \\
\hline TCri & Terneras Cría ( $<2$ meses $)$ & ITIde & Identifica Toro \\
\hline RLev & Reproductores en levante & ITPad & Identifica Toro Padre \\
\hline RSer & Reproductores en servicio & ITMad & Identifica Toro Madre \\
\hline ToCri & Terneros Cría (<2 meses) & ITFNa & Identifica Toro Fecha Nacimiento \\
\hline \multicolumn{4}{|c|}{ d. Reproducción } \\
\hline RMon & Monta Natural & PVeAd & Vermífugo Tiempo en Adultos \\
\hline RIns & Inseminación Artificial & PBaMo & Baños a animales contra Mosca \\
\hline RIat & Inseminación Artificial a Tiempo Fijo & PBBom & Baño contra Mosca con Bomba \\
\hline Ceda & Criterio incorporar Edad & PBOre & Baño contra Mosca con Orejera \\
\hline CPes & Criterio incorporar Peso & EBot & Espacio para Botiquín \\
\hline CMes & Criterio Reproducción tiempo/mes & PECarb & Enfermedad Carbón Sintomático \\
\hline CPes & Criterio Reproducción peso/Kg & PEMas & Enfermedad Mastitis \\
\hline DPrS & Días primer servicio postparto (Promedio) & PEAbo & Enfermedad Abortos \\
\hline Iep & Intervalo entre partos & PERep & Enfermedad Retención Placenta \\
\hline Spp & Servicios por preñez & PCua & Practica sanitária Cuarentena \\
\hline MCObs & Detección celo observación & PVBru & Practica Vacunación Brucella \\
\hline \multicolumn{2}{|l|}{ Salud } & PVAft & Practica Vacunación Aftosa \\
\hline PTPpc & Terneras Potrero atención parto & POLavU & Practica Ordeño Lavado de Ubre \\
\hline PTCom & Terneras Curación Ombligo & POCalA & Practica Ordeño Buena calidad del agua \\
\hline PTCal & Terneras Calostro & POSecU & Practica Ordeño Secado de Ubre \\
\hline PTTAn & Terneras Tratamiento Antibiótico & POSeUP & Practica Ordeño Secado Ubre con Papel \\
\hline PTTVa & Terneras Tratamiento Vacuna & PODesP & Practica Ordeño Desinfecta Pezones antes y después \\
\hline PTTVi & Terneras Tratamiento Vitaminas & POSelP & Practica Ordeño Sellado Pezones \\
\hline PTALi & Terneras Alojamiento libre & POPruM & Practica Ordeño Prueba Mastitis \\
\hline $\mathrm{PVe}$ & Tratamiento con Vermífugo & POLavO & Practica Ordeño Lavado de manos antes del ordeño \\
\hline PVeJo & Vermífugo Tiempo en Jóvenes & & \\
\hline \multicolumn{4}{|c|}{ e. $\quad$ Producción de leche } \\
\hline OMan & Tipo ordeño Manual & \multicolumn{2}{|c|}{ Gestión empresarial } \\
\hline $\mathrm{OMec}$ & Tipo ordeño Mecánico & $\mathrm{RNac}$ & Registros de Nacimientos \\
\hline NoOrd & Número de Ordeños/Día & RPro & Registros de Producción \\
\hline
\end{tabular}


Continuación Tabla 1

\begin{tabular}{|c|c|c|c|}
\hline OAlmC & Almacenamiento de la leche en Cantina & RRep & Registros de Reproducción \\
\hline PLLluv & Producción leche/Dia Lluvias & RCom & Registros de Compras \\
\hline PLSec & Producción leche/Día Secas & RVen & Registros de Ventas \\
\hline PLVD & Producción leche/Vaca/Día & DPInv & Decisión Propietario para Inversión \\
\hline PLTotD & Producción leche Total/Día & DPPro & Decisión Propietario para Producción \\
\hline DLAut & Leche Destino para Autoconsumo & DPUsoT & Decisión Propietario para Tecnología \\
\hline DLVent & Leche Destino para Venta & DPMer & Decisión Propietario para Mercadeo \\
\hline PLec & Precio del litro de leche & SAnas & Servicios de Análisis de Suelos \\
\hline DLEmp & Nombre de la empresa destino & SPast & Servicios de Pastos \\
\hline DLNSub & No subproductos leche & SInsA & Servicios de Inseminación Artificial \\
\hline \multicolumn{2}{|c|}{ Comercialización pecuaria } & SConR & Servicios de Control Reproductivo \\
\hline CProL & Comercializa producto Leche & SNutr & Servicios de Nutrición Animal \\
\hline CLugV & Comercialización Lugar Venta & SSani & Servicios de Sanidad Animal \\
\hline MDir & Mercadeo Directo & EServ & Entidad prestadora de servicios \\
\hline \multicolumn{4}{|c|}{ f. Mano de obra } \\
\hline MOAPerm & Mano de Obra Administrador & MVeh & Número de Vehículos \\
\hline MOOPer & Mano de Obra Ordeñador & MGua & Número de Guadañas \\
\hline \multicolumn{2}{|c|}{ Infraestructura y equipos } & MMot & Número de Motosierra \\
\hline ICas & Número de Casas & MMob & Número de Motobomba \\
\hline IEst & Número de Establos & MBom & Número de Bomba de Espalda \\
\hline Icor & Número de Corrales & MEqu & Número de Equipo de Ordeño \\
\hline ISal & Número de Saladeros & MBre & Número de Brete \\
\hline IBeb & Número de Bebederos & MMan & Número de Manguera de aspersión \\
\hline SEne & Servicio Energía & MPic & Número de Picadora \\
\hline SAcu & Servicio Acueducto & MBoE & Número de Bomba estacionaria \\
\hline \multicolumn{4}{|c|}{ g $\quad$ Información propietario } \\
\hline NProp & Nombre & PCReu & Capacitación por Reuniones \\
\hline POcup & Ocupación & PCSem & Capacitación por Seminarios \\
\hline PProc & Procedencia & PCInt & Capacitación por Internet \\
\hline PTiem & Tiempo en que vive en la región & PCPer & Capacitación por Periódico \\
\hline PTene & Tiempo en que se dedica a la ganadería & PCCel & Capacitación por Celular \\
\hline PTieF & Tiempo unidad productiva & PRCPa & Información de Pastos \\
\hline PAsoc & Pertenece a una Asociación & PRCAl & Información de Alimentación ovina \\
\hline PFede & Pertenece a una Federación & PRCAd & Información de Administración \\
\hline PSolA & Solicita Asistencia técnica Permanente & PRCRe & Información de Reproducción \\
\hline PAsisA & Solicita Asistencia Adicional & PIEFin & Recibe Ingresos exclusivamente de unidad productiva \\
\hline
\end{tabular}

Las letras corresponden al código de la variable analizada. 


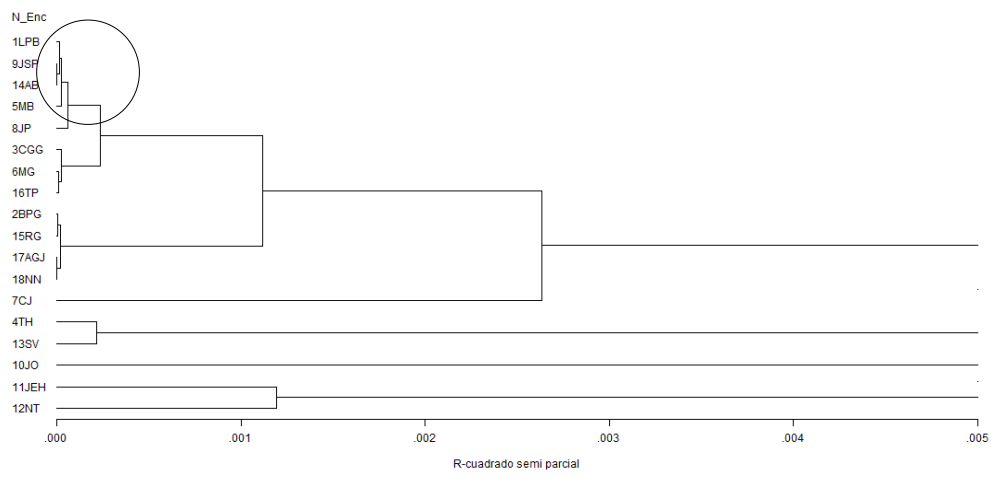

Figura 1. Dendograma de clasificación de sistemas de producción de leche en la vereda El Peñón, municipio de Sibaté (Cundinamarca). El círculo señala el clúster identificado para los sistemas de producción de leche a pequeña escala.

a)

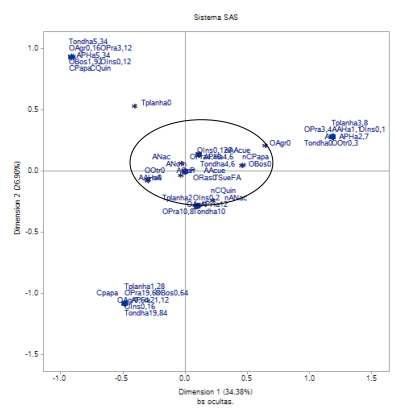

c)

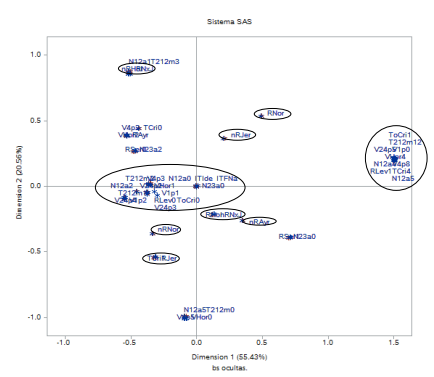

e)

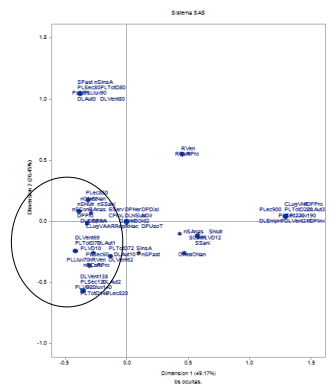

b)

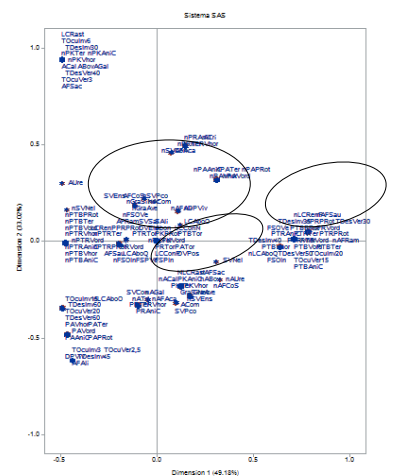

d)

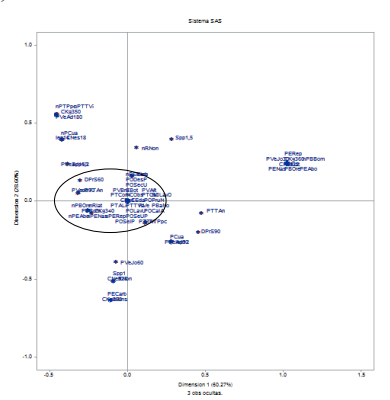

f)

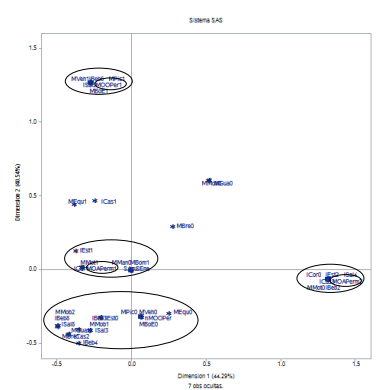

Figura 2. Análisis de correspondencia múltiple de los sistemas de producción bovina de leche en pequeña escala. (a) Localización; Aspectos biofísicos; Paisaje, uso y manejo de la tierra. (b) Recurso forrajero; Alimentación y nutrición. (c) Recurso animal. (d) Reproducción; Salud. (e) Producción de leche y destino; Comercialización pecuaria; Gestión empresarial. (f) Mano de obra; Infraestructura y equipos. 
El componente de recurso forrajero está basado mayoritariamente por praderas establecidas en especie kikuyo (Pennisetum clandestinum) (85\%), seguido por mezclas con trébol rojo (Trifolium pratense) y blanco (Trifolium repens), raygrass (Lolium hybridum), y azul orchoro (Dactylis glomerata). Como alternativa complementaria al programa de alimentación de los animales se suministra la avena (Avena sativa) como forraje de corte en forma directa y silos. De igual forma, especies arbóreas forrajeras como sauco (Sambucus nigra), acacia (Acacia Magnium), sauce (Salix humboldtiana) y aliso (Alnus acuminata) son suministradas esporádicamente en forma de ramoneo y corte; si bien, son utilizadas para la división de potreros. La proporción del área en forraje establecido con pasturas mejoradas en los sistemas analizados demuestra el grado de cambio tecnológico que han tenido en procura de una mayor productividad. Esta diversidad radica principalmente en la disponibilidad y accesibilidad de ofertas tecnológicas para los productores (CORPOICA, 1998; Murgueitio, 1999; Martínez-García, 2012; Cortez-Arriola, 2015).

Con el fin de asegurar la producción forrajera, estos sistemas realizan en su mayoría prácticas de fertilización química principalmente con úrea, triple 15 y cal y fertilización orgánica (bovinaza, gallinaza y compost) de forma ocasional. De igual forma, las labores culturales como el control de plagas y malezas y la renovación de praderas son realizadas de forma eventual. Los periodos promedios de ocupación y descanso de los potreros reportados varían según la época [invierno (48/42 días) y verano (11,1/12,8 días)], acompañado de un sistema de rotación de praderas mediante divisiones con cerca de alambre (Figura $2 b$ ).

De acuerdo a la figura $2 c$, el recurso animal presente en las unidades productivas está compuesto por diferentes biotipos raciales como: Holstein (50\%), Jersey (17\%), Ayrshire (17\%), Normando (8\%) y cruces de Holstein x Jersey (8\%) conformado por hatos promedios de 20 animales en sus diferentes etapas productivas. Para el manejo reproductivo se utilizan criterios de incorporación a la vida reproductiva como la edad (22,8 meses) y peso vivo $(334 \mathrm{Kg})$, en donde la inseminación artificial es la única biotecnología reproductiva utilizada.

En cuanto al registro de la información corresponde a registros de identificación (terneras y vacas, toro), productivos (producción de leche/vaca) y reproductivos (fecha de parto, fecha de nacimiento y número de servicios/parto), diligenciada de forma esporádica, expresado con un bajo grado de confiabilidad; por lo cual, no es reportada. Para el manejo y control sanitario de los animales, solamente se reportó la realización de prácticas sanitarias de forma preventiva (curación de ombligo, aplicación de vermífugos y baños, vacunación contra Brucelosis y Fiebre Aftosa, suministro de calostro y suplementos vitamínicos). Sin embargo, manifiestan que en la región de estudio de forma histórica hubo presencia de algunas enfermedades (carbón sintomático, mastitis, abortos y retención de placenta), aunque de forma esporádica (Figura 2d).

De acuerdo al análisis de correspondencias múltiples (Figura 2e), la producción de leche promedio por vaca/día registra un valor de 12,6 litros; encontrándose variabilidad por época seca o de lluvias. En cuanto al destino del producto lácteo, se reportó que el $97 \%$ del volumen producido es destinado para la venta directa y el restante al autoconsumo. La comercialización de leche fresca se realiza de forma directa a través de una asociación local de productores de leche $(80 \%)$ y una empresa privada (20\%); por lo cual, no se elabora ningún tipo de subproducto lácteo. Debido a los volúmenes de producción de leche reportados se evidencia la necesidad de la implementación de estrategias para el mejoramiento de la productividad y competitividad de los sistemas (Murgueitio, 1999).

Dado lo anterior, es necesario estandarizar los niveles productivos en los sistemas de producción de leche a pequeña escala, ya que la concentración de las principales industrias lecheras solamente acoge a las lecherías especializadas, las cuales son las que obtienen una mayor producción por animal y rendimiento por hectárea (Balcázar, 1992; Murgueitio, 1999; Calderón et al., 2006; FEDEGAN 2017; Ríos \& Gomez, 2008).

En el componente de gestión empresarial, se evidenció falencias en el registro de la información; sin embargo, algunos sistemas cuentan con reducida información relacionada con la producción, compras $\mathrm{y}$ ventas. Referente a las decisiones administrativas relacionadas con los planes de inversión y producción, uso de tecnología, mercadeo y distribución de ingresos están a cargo específicamente del propietario. De otra parte, se reportó que los sistemas de producción de leche a pequeña escala adquieren algún tipo de servicios (análisis de suelos y pastos, inseminación artificial y control reproductivo, nutrición y sanidad animal), principalmente a la entidad local gubernamental.

La figura 2f, describe la mano de obra utilizada en los sistemas de producción de leche, la cual reporta un númeropromediode 1,2y0,2trabajadorespermanentes/ 
año (administrador y ordeñadores, respectivamente) y un bajo número de maquinaria y equipos (vehículos, guadañas, motosierra, motobomba, bomba de espalda, brete, picadora, bomba estacionaria y equipo de ordeño), dado a su reducido nivel productivo. En cuanto a la infraestructura, estos sistemas solamente cuentan con un inventario básico como casa, establo, corral, saladeros, bebederos, necesaria para la realización de las actividades de manejo y producción animal.

Finalmente, la información suministrada por el propietario reporta que su actividad está relacionada con actividades de ganadería, independiente y empleado. De otra parte, reportan que su origen obedece a la región de estudio y que la tenencia de la tierra es propia. Igualmente, manifestaron pertenecer a una asociación y que la asistencia técnica era solicitada a través de la entidad local gubernamental.

En cuanto, a los procesos de capacitación han sido adquiridos a través de reuniones, celular e internet; sin embargo, manifiestan la necesidad de ampliar los procesos de capacitación en áreas temáticas específicas como alimentación y nutrición, reproducción y administración.

A pesar de la importante contribución de los sistemas de producción de leche a pequeña escala, pocos estudios se han realizado en Colombia con el objetivo de conocer dichos sistemas para la identificación de sus problemáticas y la evaluación de las perspectivas con miras a aumentar sus niveles productivos a nivel local y mejorar las condiciones de vida de los productores. Por estas razones, es importante ampliar el conocimiento de los sistemas de producción, con el fin de aprovechar sus características en beneficio del sector rural y de la economía en general. Ya que la actividad de la lechería a pequeña escala ha revelado su capacidad para sobrevivir en diversas condiciones económicas difíciles y generar ingresos para las familias rurales.

\section{Conclusiones}

La metodología propuesta permitió identificar cuatro grupos de sistemas de producción bovino de leche (especializada, semiespecializada, pequeña escala y familiar) ubicados en la región de estudio, de acuerdo a sus características estructurales, técnicas y productivas establecidas a través del análisis de conglomerados. La caracterización técnica-administrativa del cluster de los sistemas de producción leche a pequeña escala a través del análisis de correspondencias múltiples, permitió la identificación del modelo productivo que incluye la utilización de recursos forrajeros en praderas establecidas y pasturas mejoradas y algunas especies arbóreas para la alimentación animal. Sin embargo, eventualmente suministran silos y forrajes de corte como alternativa complementaria. En cuanto al recurso animal se incluye una diversidad de biotipos raciales y cruces especializados hacia la producción de leche. Dicho modelo reúne la aplicación rudimentaria de prácticas de manejo animal, reproductivas y sanitarias que influyen directamente sobre los indicadores productivos y reproductivos; por lo cual, es necesario articular estrategias productivas encaminadas al mejoramiento de la productividad de los sistemas y la adopción de buenas prácticas ganaderas orientadas hacia la disminución del impacto al medio ambiente. En el componente de comercialización y gestión empresarial se evidencia un limitado diseño estructural siendo relevante su acción correctiva para estrechar la brecha que existe con los sistemas especializados altamente productivos. De este estudio, se puede concluir que la identificación y caracterización de los sistemas de producción de leche a pequeña escala permite en futuras investigaciones el diseño propositivo de estrategias eficientes para ampliar la capacidad tecnológica y productiva en función de los recursos disponibles e indicadores productivos y de esta forma contribuir al fortalecimiento de la cadena productiva y el sector pecuario.

\section{Agradecimientos}

A la Universidad Santo Tomas, Vicerrectoría de Universidad Abierta y a Distancia - VUAD, Facultad de Ciencias y Tecnologías y Centro de Investigación por la financiación del proyecto. A los productores de leche de la vereda El Peñón del municipio de Sibaté (Cundinamarca-Colombia) por la información suministrada. A la Secretaria de Agricultura, Desarrollo y Ambiente adscrita a la Alcaldía Municipal de Sibaté por el contacto con los productores y apoyo logístico. 


\section{Literatura Citada}

Avilez, J.P.; Escobar, P.; von Fabeck, G.; Villagran, K.; García,

F.; Matamoros, R.; García Martínez, A.

2010. Caracterización productiva de explotaciones lecheras empleando metodología de análisis multivariado. Revista Científica, 20 (1): 74-80.

Balcázar, A.

1992. La ganadería bovina en Colombia, 1970-1991. Santafé de Bogotá. Coyuntura Agropecuaria, 9 (2): 113-138.

Betancourt, K.; Ibrahim, M.; Villanueva, C.; Vargas, B.

2005. Caracterización del manejo productivo de sistemas lecheros en la cuenca del río Bulbul de Matiguás, Matagalpa, Nicaragua. Livestock Research for Rural Development, 17 (7): \#80.

Calderón, A.; García, F.; Martínez, G.

2006. Indicadores de calidad de leches crudas en diferentes regiones de Colombia. Revista MVZ Córdoba, 11: 725-737.

Carrillo, L, B.; Moreira L.; Víctor, H.; González, V, J.

2011. Caracterización y tipificación de sistemas productivos de leche en la zona centro-sur de Chile: un análisis multivariable. Idesia (Arica), 29 (1): 71-81.

CNA,

2016. Censo Nacional Agropecuario, hay campo para todos. Departamento Administrativo Nacional de Estadística. Tomo 2. 1036p.

CORPOICA, Corporación Colombiana de Investigación Agropecuaria. 1998. Principales avances en investigación y desarrollo tecnológico por sistemas de producción pecuaria. Corpoica. Santafé de Bogotá. Colombia. 145p.

Cortez-Arriola, J.; Rossing, W. A.; Massiotti, R. D. A.; Scholberg,

J. M.; Groot, J. C.; Tittonell, P.

2015. Leverages for on-farm innovation from farm typologies? An illustration for family-based dairy farms in north-west Michoacán, Mexico. Agricultural Systems. 2015; 135: 66-76. FAO.

2002. Statistical database for Agriculture of the Food and Agriculture Organization of the United Nations (FAO). Rome. http://www.fao.org/faostat/en/\#home.

FEDEGAN,

2017. Federación Colombiana de Ganaderos. Balance y perspectivas del sector ganadero colombiano 2016-2017. Bogotá. 2-18.

FEDEGAN,

2015. El consumo per cápita de leche en Colombia. Fedegan-FNG Oficina de Investigaciones económica. Página del Ganadero Fedegan - Fondo nacional del Ganado - FNG. 277p.

Giraldo, O.F.

2008. Seguridad alimentaria y producción pecuaria campesina: el caso de la localidad rural de sumapaz. Luna Azul, (27): 49-59.

González-Echeverría, J.

2012. Situación actual y perspectivas del sector lácteo costarricense. Una visión de la Cámara Nacional de Productores de Leche. En: Memorias Congreso Nacional Lechero 2012. San Carlos, Costa Rica.
Hernández Morales, P.; Estrada-Flores, J.G.; Avilés-Nova, F.; Yong-Angel, G.; López-González, F.; Solís-Méndez, A.; CastelánOrtega, O. A.

2013. Tipificación de los sistemas campesinos de producción de leche del sur del estado de México. Universidad y ciencia, 29 (1): 19-31.

Martínez Castro, C. J.; Cotera Rivera, J.; Zavaleta, J. A.

2012. Características de la producción y comercialización de leche bovina en sistemas de doble propósito en Dobladero, Veracruz. Revista Mexicana de Agronegocios, 16 (30): 816-824.

Martínez-García, C.G.; Dorward, P.; Rehman, T.

2012. Farm and socio-economic characteristics of smallholder milk producers and their influence on technology adoption in Central Mexico. Tropical Animal Health and Production, 44: 1199-1211.

Murgueitio, E.

1999. Reconversión Ambiental y Social de la Ganadería Bovina En Colombia. Fundación CIPAV. 24p.

Murgueitio, E.; Calle, Z.

1998. Diversidad biológica en sistemas de ganadería bovina en Colombia. En: Sánchez, M.D.; Rosales Méndez, M. (eds.). Agroforestería para la producción animal en Latinoamérica. FAO. Roma, Italia. Pp. 27-46.

Ríos, G.; Gómez, L.;

2008. Análisis de costeo para un sistema de producción de lechería especializada "un acercamiento al análisis económico en ganadería de leche": estudio de caso. Dyna, 75 (155): 37-46.

Rodríguez, D.C.; Rodríguez, M. T.; Perez, 1. B.; Molina,O. M.; Varela, O. T.; Ayala, e. E.

2012. Evaluación de la sustentabilidad social, económica y productiva de dos agroecosistemas de producción de leche en pequeña escala en el municipio de Amecameca, México. Revista Cientifica UDO Agricola, 12 (3): 690-704.

Smith R., R.; Moreira L., V.; Latrille L., L..

2002. Caracterización de sistemas productivos lecheros en la x región de chile mediante análisis multivariable. Agricultura Técnica, 62 (3): 375-395.

Solano, C.; Bernués, A.; Rojas, F.; Joaquín, N.; Fernández, W.; Herrero, M.

2000. Relationships between management intensity and structural and social variables in dairy and dual-purpose systems in Santa Cruz, Bolivia. Agricultural Systems, 65: 159-177.

Solano, C.; León, H.; Pérez, E.; Herrero, M.

2003. The role of personal information sources on the decision-making process of Costa Rica dairy farmers. Agricultural Systems, 76: 3-18.

Vargas-Leitón, B.; Solís-Guzmán, O.; Sáenz-Segura, F.; LeónHidalgo, $\mathrm{H}$.

2013. Caracterización y clasificación de hatos lecheros en Costa Rica mediante análisis multivariado. Agronomía Mesoamericana, 24 (2): 257-275. 